\title{
Factores que interferem nas magnitudes de impacto na recepção ao solo no remate em suspensão do andebol
}

\author{
Interfering factors in the impact magnitudes during jump throw landing \\ in handball
}

\author{
S.G. Santos, D. Detanico, T. Piucco
}

\begin{abstract}
Este estudo objectivou analisar as magnitudes dos impactos nos membros inferiores de atletas de andebol durante a recepção ao solo no remate em suspensão e relacioná-las com massa corporal (MC) dos atletas, altura do salto (AS) e ângulos de flexão do joelho (AJ) e do quadril (AQ) na recepção. Participaram nove atletas de andebol com idade de $22.2 \pm 1.4$ anos. Foi utilizado um acelerómetro triaxial para análise dos impactos no joelho e no tornozelo e um sistema de reconstrução tridimensional de movimento para análise cinemática do AQ AJ e AS. Utilizou-se estatística descritiva, análise de variância e regressão linear múltipla, com significância de 5\%. As maiores magnitudes de impactos ocorreram no eixo antero-posterior, tanto no joelho $(28.24 \mathrm{~g})$, quanto no tornozelo $(59.81 \mathrm{~g})$; $71 \%$ do impacto no joelho pode ser explicado pelas variáveis AQ e MC; $45 \%$ do impacto no tornozelo pode ser explicado pelas variáveis AQ e AJ. Conclui-se que o ângulo de flexão do quadril foi a variável mais determinante nos valores de impactos nos membros inferiores, enquanto a altura do salto parece não ter influência, mostrando que o domínio da técnica correcta de recepção ao solo pode minimizar as sobrecargas aplicadas no organismo dos atletas.

Palavras-chave: impacto, cinemática, sobrecarga
\end{abstract}

ABSTRACT

This study aimed to analyze the impact magnitude in the lower limbs during the landing phase in handball jump throw as well as to relate the impacts with the body weight (BW), jump height (JH) and the knee flexion angle (KA) and hip flexion angle (HA) during landing. Nine handball athletes, aged $22.2 \pm 1.4$ years took part in this study. A triaxial accelerometer was used to analyze the knee and ankle impacts. Kinematics parameters were investigated using a three-dimensional system. Descriptive statistics, analysis of variance and linear multiple regression set at $5 \%$ were used. The highest impact magnitudes occurred in the anteroposterior axis of the knee $(28.24 \mathrm{~g})$ as well as in the ankle $(59.81 \mathrm{~g})$ with $71 \%$ of the knee impact explained by HA and BW while $45 \%$ of the ankle impact were explained by HA and KA. Results allow for the conclusion that hip flexion had the highest influence on the lower limb impact whereas there was no observed influence of jump height. Therefore, correct landing technique can minimize the overload applied to the athlete's joints.

Keywords: impact, kinematics, overload

Submetido: 22.05.2010 | Aceite: 31.07.2010

Saray Giovana dos Santos, Daniele Detanico e Tatiane Piucco. Laboratório de Biomecânica; Programa de PósGraduação em Educação Física; Universidade Federal de Santa Catarina, Florianópolis - SC, Brasil.

Endereço para correspondência: Daniele Detanico, Laboratório de Biomecânica, Centro de Desportos UFSC, Campus Universitário da Trindade, CEP: 88040-900, Florianópolis - SC, Brasil.

E-mail: danieledetanico@gmail.com 
Os desportos colectivos em sua maioria apresentam movimentos básicos como corrida, saltos e recepção ao solo, os quais geram cargas dinâmicas nos membros inferiores que produzem forças em diferentes sentidos. Tais forças podem prejudicar o organismo dos atletas, contribuindo para a ocorrência de lesões crónicas desportivas ou até mesmo de doenças degenerativas articulares (Voloshin, 2004).

No andebol, o remate em suspensão é um dos fundamentos mais utilizados em treinos e jogos, correspondendo a $75 \%$ de todos os remates realizados no jogo (Wagner, Kainrath, $\&$ Muller, 2008). Este pode ser caracterizado por suas fases de aproximação, impulsão, vôo, remate propriamente dito e recepção ao solo (Pori, Bon, \& Sibila, 2005). A recepção ao solo é o momento em que um ou dois membros tocam $\mathrm{o}$ solo iniciando o movimento excêntrico, caracterizando um impacto contra o solo. Alguns factores podem interferir na magnitude desses impactos no momento da recepção ao solo, como a altura em que o atleta atinge na fase de voo, a massa corporal do mesmo (Bisseling, 2008) e os ângulos de flexão das articulações dos membros inferiores durante a recepção (Devita \& Skelly, 1992).

$\mathrm{O}$ aumento da altura do salto pode resultar em maior força de reação do solo (FRS) no momento da recepção, além de maior velocidade angular e maiores sobrecargas no joelho e no tornozelo (Bisseling, 2008). Esses aspectos determinam maior absorção de energia muscular pelos músculos flexores do tornozelo e extensores do joelho, o que pode estar associado a lesões no joelho (Hewett et al., 2005) e aumento das forças nos ligamentos do joelho (Markolf, O'Neill, Jackson, \& McAllister, 2004).

Demais factores que parecem influenciar directamente nas magnitudes de impacto durante a recepção ao solo são a massa corporal do atleta e a aceleração do mesmo. Do ponto de vista mecânico, no instante da colisão contra o solo há ocorrência de um momento linear, que depende da massa do corpo e da variação da velocidade (Bisseling, 2008). Com base nesse princípio, pode-se inferir que o impacto contra o solo está directamente relacionado com a massa do corpo envolvido e com a velocidade de contato entre os corpos (indivíduo e solo), num dado intervalo de tempo, ou seja, a aceleração (derivada da velocidade).

De acordo com Devita e Skelly (1992), cerca de $75 \%$ da energia mecânica oriunda de um impacto pode ser dissipada passivamente pela musculatura, articulações e ligamentos corporais, sendo que os $25 \%$ restantes se dão por outras estruturas como o calçado, o piso, entre outros itens. Considerando este último, alguns mecanismos podem ser adotados a fim de amenizar a magnitude dos impactos contra o solo, como a utilização de materiais com propriedades físico-mecânicas que favoreçam a absorção de energia, ou ainda acções relativas ao domínio da técnica de recepção ao solo (Santos, Detanico, \& Reis, 2009). Em relação à técnica, esta pode ser realizada utilizando maior flexão das articulações dos membros inferiores (quadril, joelho e tornozelo), direccionando as forças de impacto no sentido antero-posterior e minimizando, dessa forma, a intensidade dos impactos (Prapavessis \& McNair, 1999).

Em corredores, a relação entre os ângulos de flexão dos membros inferiores e o pico de aceleração de impacto vem sendo bastante investigada. Lafortune (1985) verificou que um aumento da flexão do joelho melhora a atenuação do impacto causado pela aceleração da dorsi-flexão do pé durante a corrida. Derrick (2004) encontrou uma relação inversa entre o aumento de flexão do ângulo do joelho e o pico de aceleração em diferentes protocolos de corrida que consideravam o comprimento do passo, o tipo de piso, a intensidade e a fadiga do corredor.

Alguns estudos têm investigado as características dos impactos nos membros inferiores em diferentes modalidades como as quedas no judô (Piucco \& Santos, 2010; Santos, Melo, Heidrich, Moro, \& Reis, 2007), a 
recepção ao solo no voleibol (Santos, Esteves, Leite, Oliveira, \& Chagas, 2005) e no basquetebol (Piucco, Santos, Pacheco, Souza, \& Reis, 2007). Por outro lado, não foram encontrados estudos que investiguem quais os factores podem explicar as magnitudes de impactos em modalidades que tenham fundamentos técnicos de saltos (ataque, bloqueio, bandeja, rebote, remate em suspensão, etc), o que gera uma lacuna na literatura em relação a estes aspectos.

Desse modo, este estudo teve como objectivos: i) comparar as magnitudes de impacto advindas da recepção ao solo no remate em suspensão no andebol entre os eixos ântero-posterior, vertical e látero-lateral; e ii) relacionar os impactos com a altura do salto, a massa corporal dos atletas e os ângulos de flexão do joelho e do quadril na recepção ao solo do remate.

\section{MÉTODO}

\section{Amostra}

Participaram deste estudo descritivo correlacional nove atletas de andebol do sexo masculino (2 extremos, 2 laterais, 2 pivots e 3 centrais) de uma equipe universitária, com média de idades de $22.2 \pm 1.4$ anos, tempo de prática de $7.1 \pm 2.7$ anos, estatura de $1.82 \pm$ $.08 \mathrm{~m}$ e massa corporal (MC) de $83.2 \pm 10.1$ $\mathrm{kg}$. Os atletas foram selecionados intencionalmente obedecendo aos seguintes critérios de inclusão: 1) nível técnico no remate em suspensão; 2) tempo de prática na modalidade de no mínimo 2 anos; 3) treino semanal de no mínimo 2 vezes; 4) participação em competições oficiais de andebol no último ano; e 5) não possuir nenhum tipo de lesão. Em função desses critérios, alguns atletas da equipe universitária foram excluídos da amostra.

\section{Instrumentos e Procedimentos}

Antes de se iniciarem os procedimentos para a recolha dos dados, os atletas que participaram do estudo foram esclarecidos sobre os objectivos e os métodos da pesquisa, para assinarem o termo de consentimento livre e esclarecido. O presente estudo foi aprovado por um Comitê de Ética em Pesquisas com Seres Humanos (parecer n. ${ }^{\circ}$ 73/2007).

Os atletas realizaram um aquecimento prévio de aproximadamente 5 minutos, composto por saltos, alguns sprints e alongamentos. Posteriormente, cada atleta executou um remate em suspensão com corrida de balanço a fim de que os autores identificassem o primeiro membro que tocava o solo após a trajectória aérea. Em seguida foi fixado o acelerômetro no joelho (sobre a articulação tibio-femural) e no tornozelo (logo acima do maléolo lateral da tíbia) do primeiro membro que tocava o solo. Ainda, foram fixados marcadores reflexivos nos pontos anatômicos do acrômio, trocânter maior do fêmur, côndilo lateral do fêmur, maléolo lateral da fíbula e cabeça do segundo metatarso para análise cinemática do movimento.

\section{Aquisição das magnitudes de impacto}

Para mensurar os impactos nos membros inferiores durante a recepção ao solo foi utilizado um acelerômetro triaxial (transdutor piezoelétrico) do Tipo 4321 da Brüel \& KjaerTM, com dimensões de $28.6 \times 28.6 \times$ $17.0 \mathrm{~mm}$, com capacidade máxima de choque de $1000 \mathrm{~g}$ (aceleração da gravidade) e faixa de frequência de 0.1 a $12000 \mathrm{~Hz}$. Para a amplificação dos sinais foram utilizados três pré-amplificadores de carga, um para cada eixo ( $x$ - ântero-posterior; $y$ - látero-lateral e $z$ vertical) modelo 2635 da Brüel \& KjaerTM. Para a aquisição dos sinais foi utilizado o módulo MCS1000 da Lynx® ${ }^{\circledR}$ composto por 16 canais de até $+10 \mathrm{~V}$, processados pelo programa de aquisição de sinais AqDados 7.02 e para análise foi utilizado o programa AqDAnalysis 7.

Para as medidas dos impactos, os atletas realizaram 10 remates em suspensão com corrida de balanço, com o acelerômetro fixado no joelho e outras 10 repetições com o acelerômetro fixado no tornozelo do primeiro membro que tocava o solo, conforme 
metodologia proposta por Santos (2003). As magnitudes de impacto foram obtidas a partir dos valores de pico dos sinais (ver Figura 1), divididos por $9.81 \mathrm{~m} \cdot \mathrm{s}^{-2}$ para serem transformados em unidades de aceleração da gravidade $(g)$.

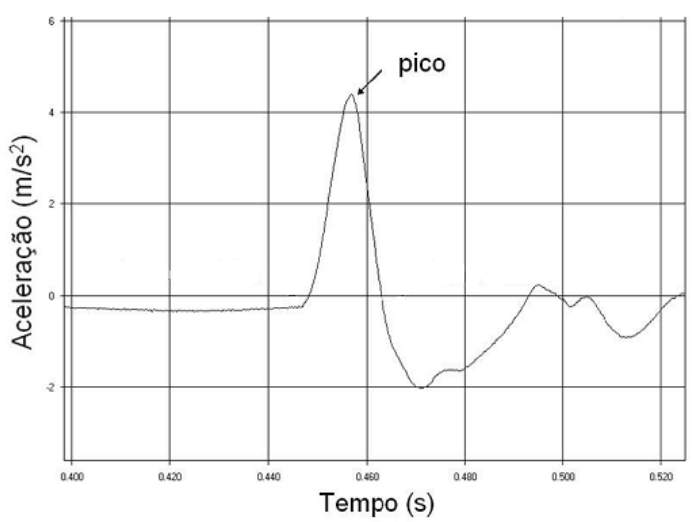

Figura 1. Critério adotado para obter o pico de impacto em cada eixo

\section{Aquisição das variáveis cinemáticas}

Para a análise cinemática foi utilizado o sistema de reconstrução tridimensional de movimento DMAS $5.0{ }^{\circledR}$ da SPICATek ${ }^{\circledR}$ composto por 3 câmeras filmadoras digitais DALSA-CCD ${ }^{\circledR}$ com frequência de aquisição de 40 fps. Os registos videográficos foram adquiridos pelo software DMAS $5.0{ }^{\circledR}$, onde as imagens foram digitalizadas para posterior reconstrução espacial do movimento. Este procedimento é realizado pelo método DLT (Direct Linear Transformation) que transforma as coordenadas bidimensionais em tridimensionais.

No total foram realizados 20 remates em suspensão em dois momentos (10 com o acelerômetro fixado no joelho e 10 fixado no tornozelo), sendo filmadas três coletas de cada momento $\left(1^{\mathrm{a}}, 5^{\mathrm{a}}\right.$ e $\left.10^{\mathrm{a}}\right)$ para posterior análise cinemática. Isso se deu em virtude de que o tempo de aquisição da análise cinemática foi maior que o tempo de aquisição da acelerometria, tendo em vista que não houve intervalo entre os remates.

As variáveis cinemáticas analisadas foram a altura do salto (AS) e os ângulos relativos de flexão do quadril (AQ) e do joelho (AJ) no momento que o primeiro membro tocava $\mathrm{O}$ solo. Para a obtenção da altura do salto, subtraiu-se a coordenada vertical, referente à articulação do quadril no momento da altura máxima alcançada, pela coordenada vertical do quadril quando o sujeito estava no solo (antes do salto).

\section{Análise Estatística}

Foi utilizada estatística descritiva (média, desvio-padrão e coeficiente de variação), análise de variância (ANOVA) com post-hoc de Tukey e análise de regressão múltipla pelo método backward, tendo as magnitudes de impacto como variável dependente e os ângulos de flexão (joelho e quadril), altura do salto e massa corporal como variáveis independentes. Foi utilizado um intervalo de confiança de $95 \%$ para a ANOVA e $80 \%$ para a inclusão de variáveis na regressão múltipla.

\section{RESULTADOS}

Na comparação das magnitudes de impacto entre os eixos ântero-posterior $(x)$, láterolateral $(y)$ e vertical $(z)$, observou-se diferença significativa entre todos os eixos, sendo que o eixo ântero-posterior apresentou os maiores valores de impacto, tanto no joelho, quanto no tornozelo (ver Tabela 1).

\section{Tabela 1}

Comparação das magnitudes de impacto no joelho e no tornozelo entre os eixos ântero-posterior (x), látero-lateral (y) e vertical $(z)$

\begin{tabular}{ccccc}
\hline Variáveis & Eixos & $M$ & $D P$ & CV (\%) \\
\hline \multirow{2}{*}{ Impacto } & $x$ & $28.24^{*}$ & 8.09 & 28.63 \\
joelho $(g)$ & $y$ & $4.90^{*}$ & 5.48 & 45.44 \\
& $z$ & $11.60^{*}$ & 5.68 & 31.02 \\
\hline \multirow{2}{*}{ Impacto } & $x$ & $59.81^{*}$ & 22.35 & 37.36 \\
tornozelo $(g)$ & $y$ & $23.08^{*}$ & 11.48 & 49.73 \\
& $z$ & $46.20^{*}$ & 24.50 & 53.04 \\
\hline
\end{tabular}

* Diferença significativa entre os eixos de cada articulação

Na Tabela 2 estão apresentados os valores descritivos das variáveis cinemáticas: ângulo de flexão do quadril e do joelho no momento de 
contato com o solo e altura máxima atingida no salto. Pode-se verificar que a altura máxima atingida no salto possui moderada variabilidade entre os atletas (15.69\%), porém os ângulos de flexão do quadril (6.96\%) e do joelho $(6.88 \%)$ demonstraram homogeneidade nas recepções ao solo.

Tabela 2

Altura máxima do salto (AS), ângulo de flexão do quadril (AQ) e ângulo de flexão do joelho (AJ) na recepção ao solo no remate

\begin{tabular}{cccc}
\hline Variáveis & AS $(\mathrm{cm})$ & AQ $\left(^{\circ}\right)$ & AJ $\left(^{\circ}\right)$ \\
\hline Média & 41.51 & 27.24 & 25.77 \\
DP & 6.51 & 10.64 & 10.60 \\
CV $(\%)$ & 15.69 & 6.96 & 6.88 \\
\hline
\end{tabular}

Na Tabela 3, observa-se que o valor de impacto no joelho (eixo $x$ ) pode ser explicado pelas variáveis AQ e MC (71\%); a magnitude de impacto no joelho (eixo $y$ ) pode ser explicada pelas variáveis AQ e AJ (21\%) e; a magnitude de impacto no joelho (eixo $z$ ) pode ser explicada pelas variáveis AQ e MC (20\%). Com relação ao impacto no tornozelo (eixo $x$ ) pode ser explicado pelas variáveis $\mathrm{AQ}$ e AJ (45\%).

Tabela 3

Regressão múltipla entre as magnitudes de impacto nos eixos $x, y$ e $z$ no joelho e no tornozelo com as variáveis $A J$, $A Q, M C$ e AS

\begin{tabular}{cccc}
\hline & Eixos & $\begin{array}{c}\text { Variáveis } \\
\text { independentes }\end{array}$ & $R^{2}$ \\
\hline Impacto & $x$ & $\mathrm{AQ}, \mathrm{MC}$ & .71 \\
joelho $(g)$ & $y$ & $\mathrm{AQ}, \mathrm{AJ}$ & .21 \\
& $z$ & $\mathrm{AQ}, \mathrm{MC}$ & .20 \\
\hline \multirow{2}{\text{Impacto}}{} & $x$ & $\mathrm{AQ}, \mathrm{AJ}$ & .45 \\
tornozelo $(g)$ & $y$ & - & - \\
& $z$ & - & - \\
\hline
\end{tabular}

É importante destacar que o AQ foi a única variável incluída para predição em todos os modelos estatisticamente significativos e a variável AS não foi incluída em nenhum modelo por não ser significativa para o nível estipulado. Da mesma forma, no impacto no tornozelo (eixos $y$ e $z$ ) nenhuma variável foi incluída, com base no critério estatístico definido.

\section{DISCUSSÃO}

No presente estudo verificou-se que as maiores magnitudes de impacto na recepção ao solo foram observadas no eixo ântero-posterior $(x)$, tanto no joelho, quanto no tornozelo, seguidas pelo eixo vertical $(y)$. Esse resultado pode ser justificado pelas características do remate em suspensão com corrida de balanço, sendo que o atleta executa o salto vertical com deslocamento horizontal e a recepção ao solo ocorre com desequilíbrio para frente, exigindo grandes solicitações tanto muscular quanto articular. Desse modo, as forças de impacto são direccionadas no sentido ântero-posterior, sendo este o movimento correto de uma recepção ao solo (Prapavessis \& McNair, 1999).

Com relação às variáveis angulares (AQ e AJ), Krosshaug, Slauterbeck, Engebretsen e Bahr (2006) encontraram valores médios de flexão do quadril de $19^{\circ}$ e do joelho de $11^{\circ}$ no momento de recepção ao solo no remate em suspensão do andebol, o que indica valores inferiores aos encontrados neste estudo. Tais resultados podem ser justificados pelo maior nível competitivo dos atletas do estudo supracitado e, consequentemente, maior aptidão neuromuscular, o que justificaria uma menor necessidade de flexionamento para sustentar a força excêntrica necessária na queda.

No voleibol, apesar de utilizar recepções diferentes do andebol, Lobietti, Fantozzi, Stagni e Merni (2006) encontraram valores angulares nos membros inferiores muito similares ao deste estudo ( $28^{\circ}$ de flexão no quadril e $19.5^{\circ}$ de flexão no joelho) durante a recepção ao solo com um pé (similar ao desta investigação) no fundamento do remate. Apesar dos valores angulares das articulações dos membros inferiores variarem de acordo com a técnica de recepção ao solo, dependente das características de cada modalidade, Devita 
e Skelly (1992) apontam que menores ângulos inter-segmentares do joelho e do quadril são capazes de absorver mais energia durante a recepção quando comparado a maiores ângulos.

Neste estudo, o aumento do ângulo de flexão do quadril foi o principal responsável pela diminuição da aceleração do impacto, tanto no tornozelo, quanto no joelho. Tais resultados divergiram de outra investigação (Decker, Torry, Wyland, Sterett, \& Steadman, 2003) que analisou diferentes modelos de recepção ao solo em atletas amadores e verificou que a flexão do joelho foi o principal responsável pela absorção da energia (amortecimento) na recepção ao solo sobre uma plataforma de força a uma altura de 60 $\mathrm{cm}$. Porém, tais diferenças podem ser justificadas devido às características de saltos e recepção ao solo diferenciadas, além de que outras variáveis que não foram controladas no presente estudo, como a velocidade angular das articulações dos membros inferiores no momento da recepção, podem ter interferido nos resultados. Ainda, Yu, Lin e Garrett (2006) sugerem que o aumento da velocidade angular de flexão do quadril e do joelho tem mostrado ser um factor determinante na diminuição do impacto, tanto no sentido vertical, como ântero-posterior.

Além do AQ outra variável que explicou as magnitudes de impacto no joelho (eixos $x$ e $z$ ) foi a MC. Do ponto de vista mecânico, tais resultados podem ser justificados pelo princípio da $2^{\mathrm{a}}$ Lei de Newton, na qual a força é dependente da aceleração na qual o corpo é submetido e da sua massa corporal. Nesse sentido, atletas com maior MC tendem a possuir maiores magnitudes de impacto nos membros inferiores durante a recepção de um salto. No entanto, essa variável não deve explicar isoladamente as forças de impacto, pois a técnica de recepção com um dos pés, representada no presente estudo pelas variáveis angulares $\mathrm{AQ}$ e $\mathrm{AJ}$, também se mostraram preditoras do impacto no joelho.
Com relação ao impacto no tornozelo (eixo $x$ ), as variáveis angulares (AQ e $\mathrm{AJ}$ ) puderam explicá-lo de maneira significativa. Para Self e Paine (2001), torna-se difícil analisar a participação desta articulação isolada no processo de recepção ao solo, mas para efeitos de estudo, deve-se analisar este segmento corporal como um todo, incluindo as articulações dos dedos. Considerando esse aspecto, os autores investigaram diferentes tipos de técnicas de recepção ao solo a uma altura de $30 \mathrm{~cm}$ e verificaram que a flexão plantar a partir da ponta dos pés parece ser mais determinante do que a flexão do joelho na atenuação do impacto. No entanto, para que o movimento de recepção seja harmonioso, todas as articulações dos membros inferiores devem flexionar-se até um ponto ideal que não exceda os limites das estruturas articulares, justificando os achados deste estudo de que as variáveis AQ e AJ podem explicar parcialmente (45\%) as magnitudes dos impactos no tornozelo. Cabe ressaltar que não foi analisado o comportamento angular do tornozelo e dos dedos, os quais possivelmente poderiam ter interferido nos resultados encontrados.

Neste estudo, apenas a variável altura do salto não foi incluída no modelo de regressão linear. Os estudos que investigam a relação entre altura do salto e as forças aplicadas nos membros inferiores nas recepções ao solo em diferentes modalidades trazem algumas controvérsias quanto aos resultados. Alguns estudos (Bisseling, 2008; Richards, Ajemian, Wiley, \& Zernicke, 1996; Salci, Kentel, Heycan, Akin, \& Korkusuz, 2004) investigaram a dinâmica das recepções ao solo de atletas profissionais de voleibol masculino e concluíram que o aumento na altura do salto causou maiores forças de impacto e uma tendência de aumento da amplitude da flexão plantar e da velocidade angular de dorsiflexão do tornozelo no contato inicial do solo. Porém, outras investigações (McNitt-Gray, 1991; Santello \& McDonagh, 1998) não encontraram variações significativas na amplitude de 
movimento articular do tornozelo em recepções de diferentes alturas.

De maneira geral, dentre as variáveis investigadas, os valores angulares do quadril e do joelho no momento da recepção ao solo, assim como a massa corporal do indivíduo possuem maior poder de explicação nos valores de impacto nos membros inferiores. Por outro lado, independente da altura do salto, a magnitude do impacto nos membros inferiores parece não sofrer alterações significativas.

\section{CONCLUSÕES}

Diante dos resultados encontrados neste estudo, pode-se concluir que as maiores magnitudes de impacto durante a recepção ao solo no remate em suspensão ocorreram no sentido ântero-posterior, tanto no joelho quanto no tornozelo. A massa corporal, os ângulos de flexão do joelho e do quadril são variáveis que melhor explicam as magnitudes de impacto no joelho da perna de recepção. No entanto, entre as mesmas variáveis, para os impactos no tornozelo, a massa corporal parece não ter contribuído. Em geral, o ângulo do quadril foi a variável que mais influenciou nos impactos gerados nos membros inferiores, enquanto a altura do salto parece não interferir nos mesmos. Por fim, para uma melhor compreensão das forças de impacto geradas durante a recepção dos saltos, sugere-se que outras variáveis sejam investigadas como o comportamento angular do tornozelo, a velocidade angular de flexão das articulações dos membros inferiores, controle da corrida de aproximação para os saltos e o tipo de calçado utilizado pelos atletas.

\section{REFERÊNCIAS}

Bisseling, R. W. (2008). Biomechanical determinants of the jumper's knee in volleyball. Tese de Doutoramento, University of Groningen, Holanda.

Decker, M. J., Torry, M. R., Wyland, D. J., Sterett, W. I., \& Steadman, J. R. (2003). Gender differences in lower extremity kinematics, kinetics and energy absorption during landing. Clinical Biomechanics, 18, 662-669.
Derrick, T. R. (2004). The effects of knee contact angle on impact forces and accelerations. Medicine $\mathcal{E}$ Science in Sports $\mathcal{E}$ Exercise, 36, 832837.

Devita, P., \& Skelly, W. A. (1992). Effect of landing stiffness on joint kinetics and energetics in the lower extremity. Medicine $\mathcal{E}$ Science in Sports $\mathcal{E}$ Exercise, 24, 108-115.

Hewett, T. E., Myer, G. D., Ford, K. R., Heidt, R. S., Colosimo, A. J., McLean, S. G., ... Succop, P. (2005). Biomechanical measures of neuromuscular control and valgus loading of the knee predict anterior cruciate ligament injury risk in female athletes: A prospective study. The American Journal of Sports Medicine, 33, 493-501.

Krosshaug, T., Slauterbeck, J. R., Engebretsen, L., \& Bahr, R. (2006). Biomechanical analysis of anterior cruciate ligament injury mechanisms: Three-dimensional motion reconstruction from video sequences. Scandinavian Journal of Medicine and Science in Sports, 10, 1-12.

Lafortune, M. A. (1985). Jumping mechanics and jumper's knee. Sports Science and Medicine, 2, 2-4.

Lobietti, R., Fantozzi, S., Stagni, R., \& Merni, F. (2006). Kinematics analysis of landing from volleyball spike followed by block: A pilot study. Gait \& Posture, 24(S1), 47-48.

Markolf, K. L., O’Neill, G., Jackson, R. S., \& McAllister, D. R. (2004). Effects of applied quadriceps and hamstrings muscle loads on forces in the anterior and posterior cruciate ligaments. The American Journal of Sports Medicine, 32, 1144-1149.

McNitt-Gray, J. L. (1991). Kinematics and impulse characteristics of drop landings from three heights. International Journal of Sports Biomechanics, 7, 201-224.

Piucco, T., \& Santos, S. G. (2010). Valores de impacto no corpo do judoca ao ser projetado pela técnica Ippon-Seoi-Nage. Motricidade, 6(1), 71-83.

Piucco, T., Santos, S. G., Pacheco, A. G., Souza, P. D., \& Reis, D. C. (2007, Maio). Magnitude dos impactos durante as aterrissagens no basquete associado com lesões nos membros inferiores. Comunicação apresentada no XII Congresso Brasileiro de Biomecânica, Rio Claro, Brasil.

Pori, P., Bon, M., \& Sibila, M. (2005). Jump shot performance in team handball: A kinematic model evaluated on the basis of expert modelling. Kinesiology, 37, 40-49. 
Prapavessis, H., \& McNair, P. J. (1999). Effects of instruction in jumping technique and experience jumping on ground reaction forces. Journal of Orthopedic Sports and Physical Therapy, 29, 352-356.

Richards, D. P., Ajemian, S. V., Wiley, J. P., \& Zernicke, R. F. (1996). Knee joint dynamics predict patellar tendinitis in elite volleyball players. The American Journal of Sports Medicine, 24, 676-683.

Salci, Y. B., Kentel, B., Heycan, C., Akin, S., \& Korkusuz, F. (2004). Comparison of landing maneuvers between male and female college volleyball players. Clinical Biomechanics, 19, 622628.

Santello, M., \& McDonagh, M. J. N. (1998). The control of timing and amplitude of EMG activity in landing movements in humans. Experimental Physiology, 83, 857-874.

Santos, S. G. (2003). Estudo das características de impacto e da percepção humana de conforto na prática de ukemis em diferentes tatamis. Tese de Doutoramento, Departamento de Engenharia de Produção, Universidade Federal de Santa Catarina, Santa Catarina, Brasil.

Santos, S. G., Detanico. D., \& Reis, D. C. (2009). Magnitudes de impacto e cinemática dos membros inferiores no arremesso em suspensão no handebol. Revista Brasileira de Cineantropometria e Desempenho Humano, 11, 326333.
Santos, S. G., Esteves, A. C., Leite, R. M., Oliveira, V. H., \& Chagas, L. (2005, Maio). Características de impacto de membros inferiores e lesões em atletas de voleibol. Comunicação apresentada no $\mathrm{X}$ Congresso Brasileiro de Biomecânica, João Pessoa, Brasil.

Santos, S. G., Melo, S. I. L., Heidrich, R. M., Moro, A. R. P., \& Reis, D. C. (2007). Aceleração e tempo de duração de impacto em segmentos corporais do judoca durante a realização de ukemi em diferentes tipos de tatames. Revista Portuguesa de Ciências do Desporto, 7, 156-166.

Self, P. B., \& Paine, D. (2001). Ankle biomechanics during the four landing techniques. Medicine $\mathcal{E}$ Science in Sports \& Exercise, 33, 1338-1344.

Voloshin, A. S. (2004). Propagação do impacto e seus efeitos sobre o corpo humano. In $\mathrm{V}$. Zatsiorsky (Ed.), Biomecânica no esporte: Performance e prevenção da lesão (pp. 452-459). Rio de Janeiro: Guanabara Koogan.

Wagner, H., Kainrath, S., \& Muller, E. (2008) Coordinative and tactical parameters of teamhandball throw: The correlation of level of performance, throwing quality and selected technique-tactical parameters. Leistungssport, 38 , 35-41.

Yu, B., Lin, C. F., \& Garrett, W. E. (2006). Lower extremity biomechanics during the landing of a stop-jump task. Clinical Biomechanics, 21, 297305.

(cc) EY-No Todo o conteúdo da revista Motricidade está licenciado sob a Creative Commons, excepto quando especificado em contrário e nos conteúdos retirados de outras fontes bibliográficas. 